\title{
Study on Plug and Play Access Control for Distributed Generation in Microgrid
}

\author{
B.Zeng \\ Guangxi Power Grid Electric Power Research Institute \\ Nanning 530023, China.
}

\author{
X.Y.Kong,Y.S. Zhang,Q.Yang, S.Y. Zhang, S.Y. Ge \\ Key Laboratory of Smart Grid of Ministry of Education \\ Tianjin University 300072 \\ Tianjin, China
}

\begin{abstract}
The "plug and play" and effective deployment of distributed generation accessible is important in further smart grid, which related to the security and stability of power system operation. A multi-agent system (MAS) based micro-grid control framework was proposed in the paper. The "plug and play" model based on MAS and power electronic technology were developed firstly, and the coordinating control strategy of microgrid was analysed. Finally some simulation studies obtained and a thought of the distributed generation "Plug and Play" in microgrid provided.
\end{abstract}

Keywords-smart grid;micro-grid;multi-Agent system;management;distributed generation;plug and play

\section{INTRODUCTION}

More and more people are interested in Distributed Generation Technology as its unique environmental protectionand efficiency. The power supply is usually smaller and closer to the users (typically below 50MW). And it can be output directly to the power grid.Small-scale power plant can supplyelectricityto nearbyload directly [1-2].

Smart grid has become the trend of the distributed power grid.Smart grid can adapt different types of distributed power, and make it easy to connect to the grid. This feature gives a serious challenge to the power grid [3].

Information interactive technology of equipment includes Information Technology and Standard of Communication. Distributed power is accessed to the grid by the inverter. IEEEP1547 makes standards for the Distributed Energy and provides a test standard.There are lots of researchesat home and abroad, such as the model proposed by literature [4].

Plug and play means that a distributed power supply does not need control and protect units but can access to the microgrid directly. A control method proposed in literature [5]allows the scattered layout of distributed power supply and does not need a high-speed communication between distributed powers. This method does not consider the problem of restoration of system voltage and frequency. Therefore,quality of the frequency of the system may not be able to guarantee when the micro-grid suffered serious disturbance.

When distributed power with the function of "plug and play" accesses to the grid, management technology will be needed. It includes the control strategy of the system change, energy management, quality of power maintenance and management etc.But because of the limit of the current standards and level of development, related technology of the inverter is difficult to implement in the short term.

In this paper,Multi-Agent System is recommended to building the control system ofmicro-grid with the function of "plug and play". And make a detailed analysis of the strategy method of each element micro-grid control. It provides a new way to optimize the operation control of micro-grid.

\section{DISTRIBUTED POWER CONTROL METHOD BASED ON MICRO-GRID FORM}

\section{A. Control Based on Multi-agent System}

Micro-grid cannot operate correctly without the stability and the perfect control system. The way of control is a difficult problem in the micro-grid research.Now, "control based on multi agent system" and "control based on the power management system" are the major method to control the micro-grid distributed power[6-8].

Micro-grid is composed of a lot of distributed energy. This hierarchical and three-dimensional control structure, together with the help of the dispatching control centre of distribution network, forms a multi-level control system with different functions which is particularly suitable for Multi agent control[6]. "Plug and use” technology can not only guarantee the access of micro power or micro-grid to distribution network, so as to meet the load demand, but also reduce the impact on micro-grid and large power grid. The use of MAS technology can coordinate and control micro-grid.

\section{B. System Access Architecture of DG "Plug and Play"}

As is seen in Figure 1, the micro-state agents accept the information of dispatching and components. While the component agents can run themselves, they can control the operation of components by themselves based on the data received. In general, through communication, each agent builds up its relation with others so that the micro-state can operate steadily and flexibly. 


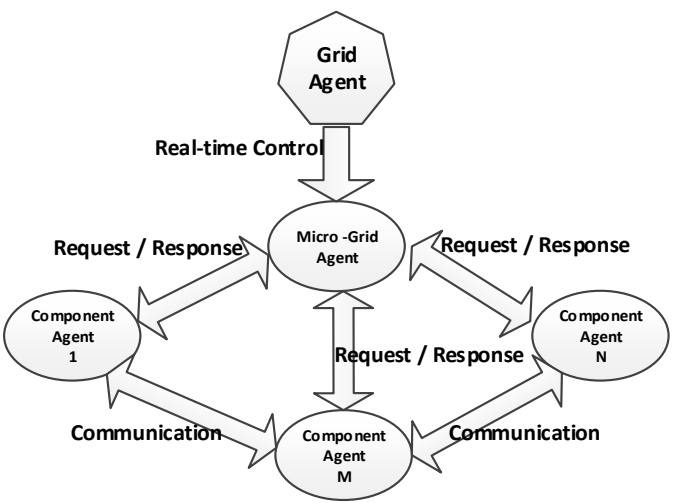

FIGURE I. STRUCTURE OF THE PLUG AND PLAY SYSTEM FOR MICRO-GRID BASED ON MAS.

Component-level agents include direct control of distributed energy, control of power generation, control of energy storage element and control of loads[7].The overall operation agent of the micro-grid is mainly for micro-grid operation in terms of internal scheduling. The centralized control of micro-grid is used here. This level is mainly used to optimize the operation of the micro-grid or just as a simple way of regional load dispatch.

Distribution network run mainly for medium-voltage network and the low voltage.These includeanumber of different typesofmicro-grid(i.e. the scheduling control of the micro-grid).This is the highestlevelagents.Specificallyhierarchyand countingare shown in Table1.

TABLE I. HIERARCHY OF MULTI-AGENT SYSTEM IN MICRO-GRID.

\begin{tabular}{|c|c|c|}
\hline Level & $\begin{array}{c}\text { Number of } \\
\text { Agents }\end{array}$ & Examples \\
\hline 1 & One & $\begin{array}{c}\text { Control, decision for single micro } \\
\text { power }\end{array}$ \\
\hline 2 & Several & $\begin{array}{c}\text { Coordinated control for the whole } \\
\text { micro-grid in the power supply and } \\
\text { load }\end{array}$ \\
\hline 3 & Several /All & $\begin{array}{c}\text { Coordinated control of the whole } \\
\text { power network }\end{array}$ \\
\hline
\end{tabular}

C. "Plug and Play" Model of Components in Micro-grid

This paper presents a new "plug and play" model. It contains three modules: First, self-determination and control module by component-agent. The second, communication and collaboration modules between components and micro-grid elements, and the last ispower electronic control system,as shown in Figure 2.

The part in virtual box represents a fundamental functional unit of component-agent. Component-agent collects operation data of micro-grid and send it to analysis processing unit. Then use the decisions command unit to make decisions. Eventually issue an order or request to ensure that the output characteristics of the respective components can meet the requirements of "plug and play" function. Also issue a request signal to other agents to inform each other if they have the need for collaboration.

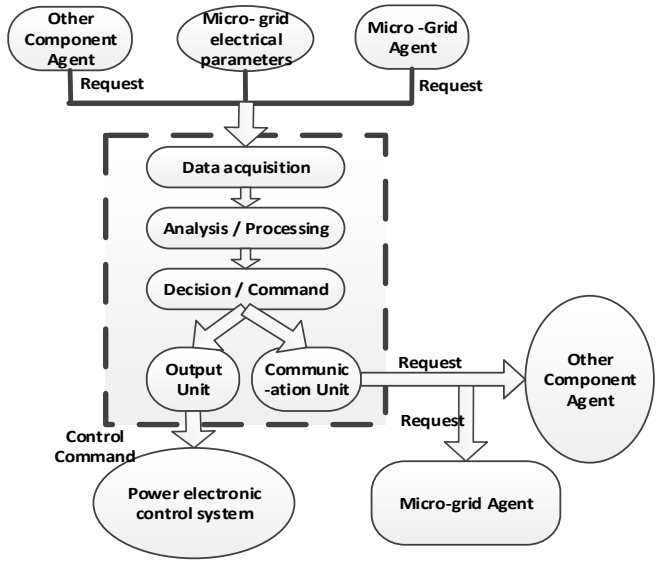

FIGURE II. "PLUG AND PLAY” MODEL OF THE COMPONENTSINMICRO-GRID.

Based on the structure above, we can classify the individual component-agent as functional agents that shown in Fig. 3. Application agents represent the various components of different internal controls.

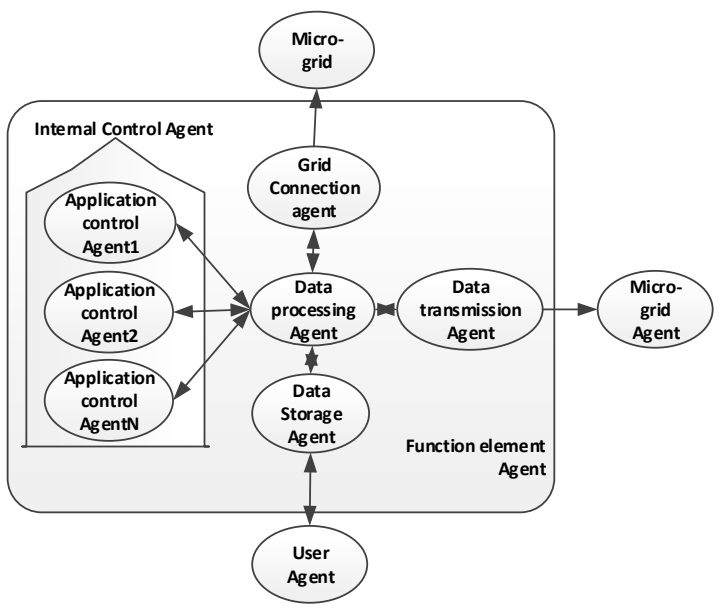

FIGURE III.

FUNCTIONS OF THE COMPONENT AGENTS IN MICRO-GRID.

\section{COORDINATED CONTROL STRATEGY OF MICRO-GRID BASED ON MAS}

The whole system has been able to operate so well especially through the mutual exchange between the agents and the cooperation to complete the assigned task. On the basis of MAS communication language and communication mechanism, we construct MAS platform based on three microgrid coordination structure.

The basic feature of a software agent is the ability of autonomy and active interaction with the surrounding environment. A Multi-Agent system is composed of many independent agents. Each of them is in close contact with each other, and also affected by environment. So for each MultiAgent System, we must not only understand the internal behaviour of each agent, you also need to analyze the factor of environment, coordination rules and mechanism from the global perspective of Multi-Agent System. In the MAS, that is 
to coordinate the relationship between each agent, such as collaborative relationship or control relationship.

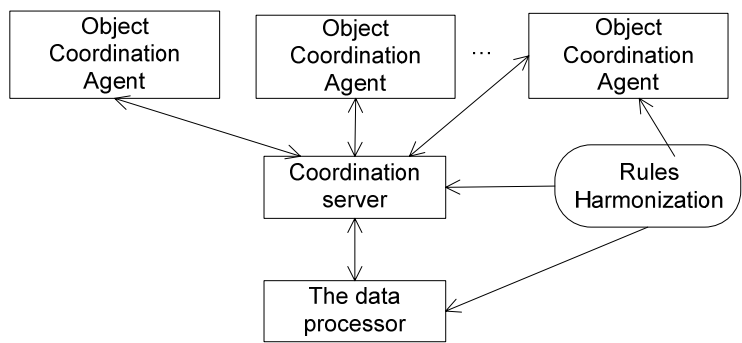

FIGURE IV. COORDINATED CONTROL STRUCTURE OF MAS

The architecture of MAS coordinated platform is shown in Figure 4. This structure is composed of $\mathrm{N}$ coordination objects (each Agent), a coordinator server, and a data processor [8].

Coordination server need receive the requests at any time, search for the destination address calling for request and send messages. Meanwhile, it also needs to establish a connection to the database and store the shared data. The data processor is used to deal with the specific information in every coordination process, including the sponsors, recipients, and coordinating content and so on, which come from the coordinating information.

We use the micro-grid as an example to discuss the coordinated control strategy of agent. At this point, the system includes micro-grid agent and component agent which also includes photovoltaic agent, battery agent and load agent. The function of each Agent are as follows:

(1) The function of micro-grid is to collect the parameters of electrical network, process data, develop strategy and send to the other agent.

(2) Photovoltaic power generation Agent uses maximum power mode and the timely adjustment of the reference data of power electronic control devices to meet the requirements of the load, and communicates with the Agents of other components in the micro-grid to obtain command of control and information of coordination.

(3) Battery Agent tracks changes of system and load, adjust the power electronic devices in time, maintain the system voltage and output power stability, and communicates with the Agents of other components in the micro-grid.

(4) The Agent of components adjusts the load power, simulates the load changes under actual conditions, and communicates with the Agents of other components in the micro-grid.

\section{THE SIMULATION RESULTS}

According to the above paper, the paper, which is based on the micro- grid PSCAD model of photovoltaic power generation unit, battery and load, uses two sets of parallel three-phase inverter as a tester to verify the results, then analyses the operating effects of this model after it is plugged in the micro-grid. The inverters tested in the model isanalyzed on the basis of controllable droop positioning and traditional droop control based on Agent, and validation of the device parameter is obtained by the small signal model

As it is shown in the figure 5 , when the frequency was lowered to the threshold due to the change of active part of the load, we can improve the active power output of the inverters by adopting the method of controllable droop positioning, and the output power returns to the rated power as a result of the adoption of traditional droop control, then frequency of the power system will rise until it reaches $50 \mathrm{~Hz}$. As it is shown in the figure 6 , when the amplitude of the joint nodes voltage drops due to the increase of reactive power, we can improve the reactive power output of the inverters by adopting the method of controllable droop positioning, and the output power returns to the rated power as a result of the adoption of traditional droop control, then amplitude of the joint nodes voltage will return to the rated voltage.

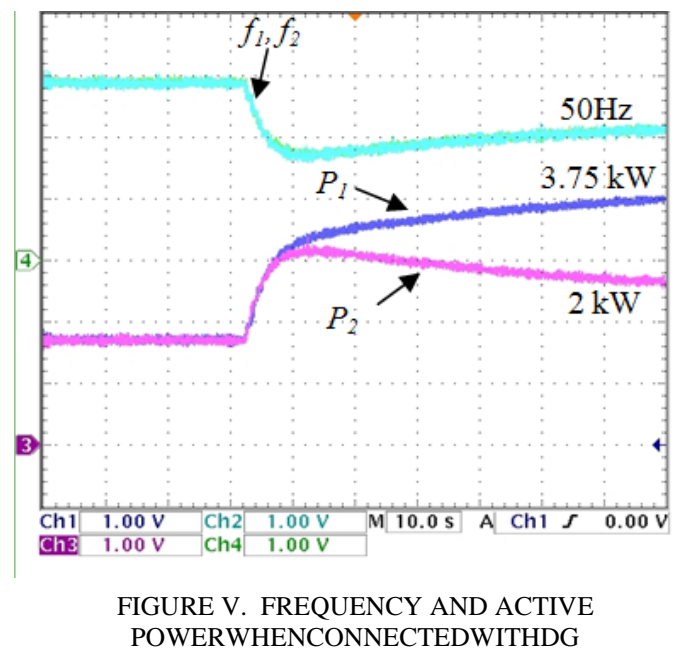

By comparison above we can find, the voltage and frequency of the micro-grid can be ensured to change in the range of what we set in advance when one of the inverters in the micro-grid is controlled in the method of controllable droop positioning. And the power output of the inverters can always be kept in the rating working point. As a result, each component can be controlled by itself according to the operation situation of the system by adopting the technology of "Plug and Play" Control based on MAS. Meanwhile, the cooperation of each component can be strengthened. Finally, these components will maintain the stable operation of the micro-grid system together.

\section{SUMMARY}

In this paper, we've analyzedthe advantage of Distributed Generation Control Method in Smart grid, and then proposed the Plug and Play control method based on multi-agent. At last, we proposed the control strategy based on PE technology and MAS.Through the simulation, we found that the control strategy on the one hand could adjust the micro-power output for the need of the load and system stabilization. On the other hand, it can control the multi-component, optimizing the micro-grid.However, because of the immaturity of DG-to-grid, there are still some problems for us to solve like the combination of DG and traditional grid and so on. 


\section{ACKNOWLEDGEMENTS}

X.Y. KONG is the corresponding author.The authors thanks for funding ofNational Science and Technology Support Program (2013BAA01B03).

\section{REFERENCES}

[1] Y. Ji, Q. Ai, D.Xie. Research on Co-Developmental Trend of Distributed Generation and Smart Grid, Power System Technology,2010,34(12):1523.

[2] Y. Luo, L. Shi, G.Y. Tu. Microgrid Common Information Model Applicable to Plug and Play Feature of Distributed Resources,Automation of Electric Power System,2010, 34(8):97-100.

[3] L.Y. Kang, H.X. Guo, J. Wu. Characteristics of Distributed Generation System and Related Research Issues Caused by Connecting It to Power System, Power System Technology,2010， 34(12):43-49.

[4] S.H. Zhou, W. Pei, Z.P. Qi. Common Interface for Interconnection Between Distributed Generation and Microgrid, Automation of Electric Power System,2010,34(3):91-95.

[5] NIKKHAJOEI H, LASSETER R H. Distributed generation interface to the CERTS microgrid, IEEE Trans on Power Delivery, 2009, 24 (3): 1598-1608.

[6] Katiraei F, Iravani M R. Power management strategies for a microgrid with multiple distributed generation units, IEEE Transactions on Power Systems, 2006,21(4): 1821-1831.

[7] J.H. Guo, Q.H. Xie, W. Huang. Study on the Plug and Play Theory in Micro-grid Based on MAS, Power System and Clean Energy,2010,26(1):31-35.

[8] J. Zhang, Q. Ai, X.G. Wang. Application of Mult-i agent System in a Microgrid, Automation of Electric Power System,2008， 32(24):80-87. 